\title{
VOIR ET SEE : ÉTUDE COMPARÉE DE LA CONSTRUCTION A ATTRIBUT DE L'OBJET ${ }^{1}$
}

\author{
Christelle LACASSAIN-LAGOIN
}

Université de Pau et des Pays de l'Adour, Pau (France)

Centre de Recherche en Poétique, Histoire Littéraire et Linguistique, EA $3003^{2}$

\begin{abstract}
En): This paper sets out to compare the meaning and uses of the visual perception verbs see in English, and voir in French, in object-oriented predicative complement constructions. It is aimed at identifying how such sentences function both syntactically and semantically and how the verb and the complements interact, trying in particular to determine the structure of socalled object-oriented complement structures. This qualitative, corpus-based study focuses on three categories of complements - adjective phrases, preposition phrases and noun phrases. It shows that the two verbs license complements that are syntactically similar. The analysis brings to light three main types of semantic reading - visual perception, perceptual inference and cognition - and demonstrates that, for each of them, the meaning of the sentences is construed with the help of three crucial factors: the object referent and its ontological nature; the syntactic category of the predicative complement and its interaction with the object; the semantic compatibility between the predicative complement and the perception verb. It also appears that voir has a wider range of uses in the domain of cognition while see seems to have a larger range in the domain of both visual perception and perceptual inference.
\end{abstract}

Keywords (En): perception verbs; see; voir; object-oriented predicative complement; visual perception; perceptual inference; cognition

Mots-clés (Fr) : verbes de perception ; see ; voir ; attribut de l'objet ; perception visuelle ; inférence perceptuelle ; cognition

\section{Introduction}

L'objectif de cette étude est de comparer, dans une perspective énonciativiste et cognitiviste, les verbes de perception visuelle see en anglais (langue germanique) et voir en français (langue romane) dans des constructions pour lesquelles il est traditionnellement dit que le complément d'objet du verbe est suivi d'un attribut de l'objet. L'analyse se concentre sur les attributs adjectivaux, nominaux et prépositionnels, et tente d'apporter une réponse à deux questions principales :

1) En lien avec le concept de coprédication, peut-on considérer que l'attribut de l'objet (le syntagme adjectival alive and well / mort en (1-2)) constitue une idée seconde par rapport à une idée première (she saw Eleanor / d'aucuns aimeraient le voir)?

2) Quels sont les liens entre syntaxe et sémantique dans les constructions à attribut de l'objet ? On s'interrogera sur la connexion ou déconnexion entre syntaxe et sémantique dans les énoncés comportant une construction à attribut de l'objet, afin de déterminer s'il existe une relation entre la catégorie grammaticale de l'attribut et le sens véhiculé par l'énoncé.

\footnotetext{
${ }^{1}$ Nous tenons à remercier les relecteurs pour leurs remarques pertinentes et leurs conseils avisés.

${ }^{2}$ La charte de signature des publications de l'UPPA indique cette appellation précise : UNIV PAU \& PAYS ADOUR, Centre de Recherche en Poétique, Histoire Littéraire et Linguistique, EA3003, 64000, Pau, France.
} 
(1) If she saw Eleanor alive and well it might frighten her into some sort of submission. $(B N C)$

(2) D'aucuns, parmi ses marchands, aimeraient le voir $\underline{\text { mort }}$; certains critiques aussi. (Lextutor)

Il s'agit, dans un premier temps, de poser les particularités de see et voir et de justifier le choix de leur traitement. Sont ensuite présentées les caractéristiques des constructions à attribut de l'objet, ainsi que leurs similitudes et différences avec les structures transitives "simples". Puis l'étude procède à une comparaison des trois types d'attributs de l'objet, notamment de leur distribution et des nuances sémantiques que chacun apporte à l'énoncé. Elle propose, pour finir, une synthèse sur les trois configurations étudiées et leurs emplois avec les deux verbes de perception en question.

L'analyse repose sur des énoncés authentiques extraits de cinq corpus (voir le détail dans la bibliographie), et l'approche adoptée y est qualitative plutôt que quantitative, car l'intérêt est principalement centré sur les différences sémantiques véhiculées par des énoncés comportant une construction syntaxique donnée, acceptée par deux verbes appartenant à deux langues différentes.

\section{Verbes et construction sélectionnés pour l'étude}

Les verbes see et voir s'organisent autour de l'axe non agentif ${ }^{3}$ du champ de la modalité visuelle: ils renvoient à un procès borné, à un achèvement ${ }^{4}$, à un phénomène ponctuel dans le réel, où un stimulus s'impose dans le champ perceptif du percevant, expérient de ce procès ${ }^{5}$. En disant I see / Je vois, l'énonciateur localise dans son expérience une perception qui l'affecte; en cela, les verbes de perception sont des localisateurs (CULIOLI, $1990: 206$ ). Les verbes de perception non agentive ou «private verbs" (PALMER, 1988) figurent dans des énoncés qui traduisent l'inchoation du phénomène perceptif et l'absence de préconstruction du complément. En effet, on est maître de ce que l'on regarde, mais non de ce que l'on voit ; cela reflète le caractère indissociable du visible et du vu.

Le choix de see et voir pour cette étude des constructions à attribut de l'objet s'est imposé pour plusieurs raisons. Tout d'abord, les unités verbales qui expriment une perception par des organes sensoriels distaux (la vue, l'audition et le toucher, respectivement), par opposition à celles qui expriment une perception par des organes sensoriels proximaux (l'odorat et le goût), présentent un éventail étendu de constructions et de significations (LACASSAIN-LAGOIN, 2007). Ainsi les verbes des modalités olfactive et gustative smell et taste, qui n'acceptent la configuration à attribut de l'objet que dans de rares cas, ont-ils été exclus d'emblée. Il en va de

\footnotetext{
${ }^{3}$ Nous préférons l'expression "perception non agentive" à celle de "perception involontaire" ou "perception passive". En effet, le percevant n'est jamais totalement inactif dans le processus de perception : il est au minimum réceptif au monde qui l'entoure, car il a la faculté d'appréhender une entité ou un événement qui surgit dans son champ perceptif.

${ }^{4} \mathrm{Si}$ see et voir dénotent souvent un procès de type achèvement, ils posent cependant des problèmes de catégorisation selon le contexte dans lequel ils apparaissent. Voir, à ce sujet, VENDLER (1967 [1957]). ${ }^{5}$ L'expérient constitue le siège subjectif d'une expérience psychologique. Cet état de fait est souligné par la définition que GrOUSSIER et RIVIÈRE (1996: 182) soumettent pour le siège : «Actant principal d'un procès du type /état/ définissable comme le repère de l'occurrence de l'état ».
} 
même pour les verbes de perception agentive. A titre d'illustration, dans la modalité visuelle, si look en anglais accepte cette configuration, ce n'est pas le cas de watch; et en français, regarder n'accepte qu'une structure en comme ${ }^{6}$. En outre, ce sont see et voir, considérés comme les verbes prototypiques de la modalité visuelle, qui ont la gamme de significations la plus large : de la perception physiologique ${ }^{7}$ à la cognition, en passant par l'imagination, l'inférence, l'opinion et le jugement. D'ailleurs, la configuration à attribut de l'objet présente un excellent échantillon de toutes ces significations.

En effet, dans certains énoncés à attribut de l'objet, les verbes de perception sont sémantiquement proches des verbes de cognition (LACASSAIN-LAGOIN, 2011), qui appartiennent également au nombre restreint des catégories verbales sémantiques qui acceptent une telle construction. Dans la typologie des prédications secondes proposée par HAVU et PIERRARD (2008b : 8-10), ces deux types de verbes font partie de la catégorie des "prédications associées à certaines fonctions syntaxosémantiques ».

Il est à noter que la perception tactile ou proprioceptive aurait pu constituer un bon candidat pour cette étude. Cependant, là où l'anglais n'offre qu'une seule unité verbale - feel, qui recouvre lui aussi une grande diversité de significations, le français possède deux verbes, sentir et ressentir, qui apparaissent également dans d'autres contextes : sentir peut aussi exprimer la modalité olfactive, et ressentir n'a pas la faculté de prendre des connotations inférentielles ou cognitives.

\section{Caractérisation syntaxique et sémantique de la construction à attribut de l'objet}

\subsection{Définition et propriétés de l'attribut de l'objet}

L'attribut, qui appartient à la catégorie des compléments, constitue un élément indispensable à l'organisation syntaxique et/ou sémantique d'un énoncé, dans la mesure où il apporte une information sur le sujet ou l'objet. Sa fonction primaire est de décrire ou d'identifier le sujet ou l'objet du verbe recteur.

1) C'est le nom donné au complément soit adjectival, soit nominal d'un verbe copulatif. P. ex. : Pierre est mon frère / Peter is my brother. Pierre devint pâle / Peter went pale. Dans ce cas, on parle d'attribut du sujet.

2) On appelle attribut de l'objet un complément analogue qualifiant un $\mathrm{C}_{1}$ et pouvant être considéré, selon les cas, comme introduit ou non par un verbe "être" effacé : Pierre rendit la vie impossible à sa famille / Peter made life (be) absolute hell for his family. (GROUSSIER \& RIVIERE, $1996: 22)^{8}$

\footnotetext{
${ }^{6}$ On trouve ainsi, pour look et regarder respectivement, des exemples tels que : As a society, we are already driven by money as the sole indicator of success and look at education as only a means to a bigger paycheck. $(C O C A) ;$ La comtesse méprisait souverainement son frère; elle le regardait comme un sot triste, et qui serait méchant si jamais il en avait le pouvoir. (Lextutor)

${ }^{7}$ L'expression «perception physiologique » est utilisée pour qualifier les énoncés qui expriment une appréhension concrète du réel, et qui se distinguent de ceux véhiculant une appréhension visuelle combinée à un processus mental, d'inférence, de jugement, etc.

${ }^{8}$ Dans la Théorie des Opérations Prédicatives et Énonciatives (TOPE), $\mathrm{C}_{1}$ symbolise le complément de rang 1 , qui correspond au complément d'objet ; il s'agit de l'élément qui renvoie au $2^{\mathrm{e}}$ argument de
} 
Ainsi, on considère souvent que l'attribut de l'objet entretient avec l'objet le même type de relation que l'attribut du sujet avec le sujet :

Il [l'attribut de l'objet] entretient avec le complément d'objet $\mathrm{N}_{1}$ le même rapport qu'un a.s. [attribut du sujet] avec le sujet dans la phrase correspondante $\mathrm{N}_{1}-\hat{e} t r e-\mathrm{X}$, ce qui conduit à considérer que, dans ces constructions, la séquence $\mathrm{N}_{1}-\mathrm{X}$ fonctionne comme une proposition réduite. Ainsi, la phrase Il a trouvé ton projet irréaliste établit entre le GN postverbal et son attribut une relation paraphrasable par Ton projet est irréaliste. (RIEGEL, PELlat \& RiOUL, 1994 : 430).

La fonction syntaxique d'attribut de l'objet peut être instanciée par des éléments appartenant à différentes catégories grammaticales. Ainsi, WILLEMS et DEFRANCQ (2000: 7) avancent qu'en français, un attribut de l'objet peut correspondre à un syntagme adjectival, nominal et prépositionnel, mais aussi à une forme verbale (ex. infinitif, participe présent) ou phrastique (relative de perception). Nous formulons donc le postulat qu'il existe des attributs prototypiques, syntagmes nominaux et adjectivaux (voir la citation de Groussier et Rivière ci-dessus, par exemple), ainsi que des attributs non prototypiques, tels que des syntagmes prépositionnels ou adverbiaux. A l'instar de LYONS (1968), mais aussi de QUIRK, GREENBAUM, LEECH et SVARTVIK (1985) ${ }^{9}$, nous estimons que ces types de syntagmes relèvent bien de la classe des compléments, et non de celle des circonstants (LACASSAIN-LAGOIN, 2011), dans les constructions à attribut de l'objet.

Pour PIERRARD et HAVU (2014: 46), l'attribut entretient avec le syntagme nominal objet un « rapport d'association » qui est typique des adjectifs - bien qu'il ne soit pas restreint à cette seule catégorie grammaticale - et identique à celui instauré par l'attribut du sujet. Les auteurs qualifient les adjectifs, les participes présents et les participes passés de « formes adjectivantes » (2014:46), appellation qui pourrait être étendue pour couvrir également les syntagmes nominaux et prépositionnels attributs de l'objet. L'attribut de l'objet modifie « la référence d'un $\mathrm{N}$ en lui attribuant "de l'extérieur" une propriété ou une qualité additionnelle », c'est-à-dire « en étendant sa description au moyen de l'attribution de propriétés par le biais de l'instauration d'une prédication [qui lui est] "extérieure" » $(2014: 32)$. Ils partagent ainsi la position de CROFT (1991), qui donne une définition approchante du rapport entre l'attribut de l'objet et le syntagme nominal auquel il se rapporte: "non restrictive modification provides a secondary comment (predication) on the head that it modifies, in addition to the main predication " (CROFT, 1991 : 52, également cité par PIERRARD \& HAVU, 2014 : 32).

\footnotetext{
la relation prédiquée. Ce terme permet de référer aux constituants nominaux et prépositionnels objets (GROUSSIER \& RIVIERE, $1996: 29$ ).

${ }^{9}$ LyONS (1968: 345-346) assimile les syntagmes prépositionnels à la droite de la copule be à des "predicative complements », et non à des adjoints, car ils ont alors un comportement identique aux syntagmes adjectivaux ou nominaux dans une configuration identique. Quant à QuIRK et al. (1985:1174), ils affirment que, lorsque les syntagmes prépositionnels sont des «obligatory adverbials », ce ne sont pas des circonstants mais des "predication adjuncts », terme qui englobe également les adverbes au fonctionnement identique.
} 
Ces définitions et/ou caractéristiques soulèvent deux questions. Peut-on faire le postulat d'une relation implicite avec la copule be / être dans les énoncés avec attribut de l'objet? S'il n'existe, pour la construction à attribut de l'objet, qu'une seule relation, d'association en l'occurrence, cela implique-t-il qu'il n'existe également qu'une seule interprétation syntaxique et/ou sémantique?

\subsection{L'attribut de l'objet : un élément facultatif ou obligatoire ?}

L'attribut de l'objet est parfois considéré comme un complément d'information qui n'est pas nécessaire à la structure syntaxique de l'énoncé. Sans doute est-ce dû au fait que, bien qu'il caractérise l'objet de la même façon qu'un attribut qualifie le sujet, il dépend moins directement du verbe recteur qu'un objet.

PIERRARD et HAVU (2014: 41) avancent que, contrairement à une construction avec adjoint, le degré d'autonomie de la coprédication par attribut de l'objet est à mettre en relation avec son degré d'implication dans la prédication régissante [PRED1]. Ils différencient alors les attributs de l'objet occasionnels (AOO), tels qu'en (3), des attributs de l'objet à double complémentation (AODC), tels qu'en (4), qui se distinguent au niveau du type d'implication dans l'énoncé : avec les AODC, l'implication est cruciale pour la grammaticalité de l'énoncé tandis qu'avec les AOO, l'implication est cruciale pour la structure informationnelle de l'énoncé.

b) les AOO : l'autonomie du co-prédicat envers PRED1 est plus restreinte dans la mesure où il influe sur la structure informationnelle de l'énoncé, sans toutefois limiter l'autonomie syntaxique du verbe nucléaire. Le co-prédicat fait partie de la zone périphérique de la microsyntaxe : [...] [ $\mathrm{V}_{\text {nucl }}-[\mathrm{TH}$ OD - co-préd]]. [...]

c) les AODC : l'autonomie du co-prédicat envers PRED1 est restreinte dans la mesure où sa présence détermine la grammaticalité de l'énoncé global. En outre, il y a une tendance à la coalescence entre le co-prédicat et le verbe nucléaire de sorte que le thème objet se rapporte au prédicat complexe qui est ainsi constitué. Le co-prédicat se situe alors dans la zone cellulaire de la micro-syntaxe : [...] [[V $\mathrm{V}_{\text {nucl }}$ - co-préd] - THod.${ }^{10}$ (PIERRARD \& HAVU, $2014: 45$ )

(3) Tu bois ton café chaud.

(4) Il estime la mesure $\underline{\text { utile. }} .{ }^{11}$

Si l'on supprime l'attribut de l'objet dans les énoncés comportant see et voir, on observe trois types de changement :

- L'absence d'un attribut de l'objet en (5a'-b') génère des énoncés au sens légèrement différent des énoncés d'origine (5a-b). L'attribut de l'objet semble être ici une expansion, et non un « argument global », de la prédication régissante (HAVU $\&$ PIERRARD, 2008a).

- En (6a'-b'), l'absence de l'attribut prépositionnel altère le sens de l'énoncé, voire sa grammaticalité : on passe ainsi d'un jugement appréciatif, dans lequel on pourrait substituer considering / considérait à seeing / voyait, à de la perception physiologique.

10 « THOD » symbolise, dans la représentation, le thème objet.

${ }^{11}$ Les exemples (3-4) sont empruntés à Pierrard et Havu (2014 : 39), bien qu'ils soient fréquemment utilisés dans la littérature sur la coprédication ou la prédication seconde. 
- De plus, en (6a), le référent de l'objet (time) ne possède pas les propriétés pour être perçu en lui-même, d'où l'acceptabilité douteuse de (6a'), pragmatiquement inconcevable.

(5a) He liked seeing Edith happy. He thought a little wistfully of Provence, tried and failed to see Edith as happy in the Hotel Paradis, and returned to savour anew the pleasures of Ramsgate. $(B N C)^{12}$

(5b) Comme il vit Chéri fort triste, il lui demanda le sujet de son chagrin. (Lextutor)

(5a') \# He liked seeing Edith. He [...] failed to see Edith. ${ }^{13}$

(5b') \# Comme il vit Chéri, il lui demanda le sujet de son chagrin.

(6a) Augustine presents a radical turn when he stops seeing time as a mark of change in $\underline{\text { nature }}$ and begins to see time as a mode of human perception. (COCA)

(6b) Elle s'imaginait elle-même en avoir un, du même genre, mais plus libre, " senza rigore ", aimait-elle à dire. Elle se voyait ainsi comme une espèce de Lespinasse et croyait avoir fondé un salon rival en enlevant à la du Deffant du petit groupe ses hommes les plus agréables, en particulier Swann [...]. (Lextutor)

(6a') ?? he stops seeing time and begins to see time.

(6b') ?? Elle se voyait.

Des configurations différentes doivent-elles être avancées pour les verbes de perception en (5) et (6) ? Lorsque la présence d'un attribut de l'objet n'influe pas sur le sens initial du verbe recteur, comme en (5), nous estimons que l'attribut est occasionnel, ce que Riegel et al. dénomment une «construction à élargissement attributif » (2014:431); c'est également le point de vue adopté par MULLER (2000), pour qui l'attribut n'est pas un élément inhérent de la valence du verbe principal. RIEGEL et al. notent d'ailleurs qu'avec un " élargissement par élément prédicatif portant sur le GN postverbal qui constitue un véritable c.o.d», qui concerne un grand nombre de verbes transitifs, le message principal de l'énoncé est constitué par le propos exprimé par l'attribut de l'objet, dont la caractérisation apporte des effets de sens variés : «état de l'objet dans une circonstance donnée [...], datation d'un événement [...], état résultatif de l'objet à l'issue d'un procès [...], etc. » (2014:431). Ainsi, en (5), happy / fort triste exprime l'état du référent de Edith / Chéri au moment de l'appréhension visuelle. Inversement, lorsque l'omission de l'attribut de l'objet rend l'énoncé agrammatical ou lui confère une signification différente, nous avons affaire à des attributs dits à double complémentation, que Riegel et al. appellent les «attributs propositionnels (ou complétifs)» (2014: 431-432).

Cela nous conduit à examiner le statut syntaxique de l'ensemble de ce qui est à droite du verbe de perception.

\footnotetext{
${ }^{12}$ Dans les énoncés étudiés, le verbe recteur est en gras, l'objet est souligné et l'attribut de l'objet est également souligné, en italiques. D'autres éléments pertinents ou de comparaison peuvent également être en italiques.

13 * signale un énoncé agrammatical ou irrecevable en contexte, tandis que \# indique un énoncé grammatical mais de sens différent ou apragmatique ; quant à ?(?), c'est le signe d'un énoncé peu recevable ou peu acceptable.
} 


\subsection{Quelle structure pour les configurations dites à attribut de l'objet ?}

Le statut de ce qui suit le verbe de perception dans les configurations à l'étude - mais également dans d'autres types de construction - est sujet à débat depuis des années.

Pour certains linguistes, il s'agit d'une proposition réduite (ou small clause) de type attributif dans laquelle le syntagme nominal post-verbal est le sujet sémantique d'un verbe be / être non exprimé ; ainsi, en (5a), Edith est considéré comme le sujet d'un be effacé, et happy comme l'attribut de ce sujet. La proposition réduite (ex. Edith happy), qui est le véritable objet du verbe recteur, alterne avec une complétive (5a"-b") comme complément du verbe principal (voir, par exemple, RIEGEL et al, 2014 : 430-432).

(5a") \# He liked seeing that Edith was happy. He [...] failed to see that Edith was as happy in the Hotel Paradis, [...].

(5a"') \# ? He liked seeing Edith who was happy. He [...] failed to see Edith who was as happy in the Hotel Paradis, [...].

(5b") \# Comme il vit que Chéri était fort triste, il lui demanda le sujet de son chagrin.

(5b"') \# ? Comme il vit Chéri qui était fort triste, il lui demanda le sujet de son chagrin.

Si les énoncés en (5) avec attribut adjectival signalent une perception physiologique, les manipulations avec une complétive en that (5a") ou que (5b") font basculer vers une lecture d'inférence perceptuelle, voire de cognition (en fonction du contexte). L'équivalence entre attribut de l'objet et complétive n'est donc pas tenable pour les verbes de perception ${ }^{14}$. Par ailleurs, les verbes de perception qui acceptent une configuration à attribut de l'objet ne régissent pas tous une complétive objet; c'est le cas, par exemple, de look et de regarder.

Cette analyse de proposition réduite est remise en question par d'autres linguistes (ex. Williams, 1983). PierRard et HAVU (2014: 34) soulignent que l'absence de be copule provoque «un certain démembrement de l'entité propositionnelle, une "dépropositionnalisation" de celle-ci par la désolidarisation de ses composantes ", et adoptent une analyse syntaxique qui permet de ne pas postuler un rapport avec une copule sous-jacente. En outre, WILLEMS \& DEFRANCQ (2000) estiment qu'on ne peut pas généraliser une relation privilégiée entre une structure à attribut de l'objet et une structure phrastique complétive, notamment dans le cas des verbes de perception, et considèrent que le $\mathrm{SN}$ à droite du verbe recteur est son objet (ex. Chéri en (5b)). L'attribut de l'objet (fort triste) correspond alors à un prédicat secondaire qui entretient une relation copulative avec l'argument objet de la prédication primaire. Cet attribut dépend donc, de manière indirecte, du verbe supérieur. Ainsi, AARTS (1995: 75) définit les prédicats secondaires comme suit: «[...] phrases which are predicated of a constituent which stands in a thematic relation to the main verb of the sentence in which the secondary predicate occurs, typically a direct object. » De la même façon, GUIMIER (1998) avance qu'un attribut adjectival est syntaxiquement optionnel et qu'il «s'ajoute à la sémantique de la

14 D'autres tests syntaxiques montrent également que l'interprétation de small clause n'est pas appropriée, tels que, par exemple, l'impossibilité de l'extraction du constituant SN objet + attribut dans une construction clivée. 
construction transitive sans la modifier $\rangle^{15}$; au niveau discursif, cependant, les deux prédications sont conçues sur le même plan énonciatif. QUIRK et al. (1985 : 11951203) postulent, quant à eux, qu'on a affaire à un schéma de complémentation «transitif-complexe » : les deux éléments à droite du verbe sont notionnellement égaux au sujet et à la prédication d'une proposition, mais ils n'agissent pas comme un seul constituant, comme le montre le test de la passivation où l'objet de la structure active est séparé de son complément. Ils incluent également les propositions non finies dans ce schéma de complémentation, estimant que celles-ci sont des attributs de l'objet non prototypiques. DECLERCK (1981), pour sa part, propose pour de telles structures une analyse en [NP + pseudo-modifier], dans laquelle l'élément qui suit le syntagme nominal - qu'il s'agisse d'un syntagme adjectival, adverbial, prépositionnel ou encore d'une proposition en V-ING (voir aussi, à ce sujet, DECLERCK, $1982^{16}$ ) - est un pseudo-modifieur : l'ensemble se comporte comme un constituant unique, mais ce qui vient à la droite du syntagme nominal diffère d'un véritable modifieur tel qu'une relative en ce qu'il peut s'ajouter à un nom-tête dont la référence a déjà été établie (un nom propre, par exemple). Ainsi, le pseudo-modifieur ne modifie pas le nom-tête au niveau sémantique, et dérive syntaxiquement d'un constituant phrastique auquel ce nom-tête appartient également. C'est la raison pour laquelle les paraphrases en (5a"'-b" ; 5a" '-b"'), qui comportent respectivement une complétive à forme finie et une relative, semblent tout autant appropriées qu'inexactes.

Nous avançons que dans une configuration dite à attribut de l'objet, un verbe de perception prend deux arguments sémantiques : un expérient et un stimulus ; ce qui est appréhendé n'est pas uniquement une entité, mais aussi un événement, une situation ou un état de fait dans lequel cette entité est partie prenante. Selon nous, une construction à attribut de l'objet, contrairement à une construction avec complétive, est en discours le reflet qu'une entité est saillante dans le procès appréhendé. Une lecture transitive-complexe ou encore une interprétation en termes de pseudo-modifieur met ainsi en valeur cette entité cognitivement saillante, tout en étant peut-être la trace d'une légère déconnexion entre syntaxe et sémantique, mais aussi entre organisation syntaxique et processus cognitif. En effet, la coprédication par attribut de l'objet est au niveau cognitif pensée en même temps que la prédication dite principale ; en (6b), par exemple, on ne "perçoit" pas le référent de time dans un premier temps, pour ensuite lui attribuer la qualification dénotée par le syntagme en as. Le référent de he considère que time is a mode of human perception : il y a une unité de perception et de caractérisation au niveau cognitif.

\footnotetext{
${ }^{15}$ Dans son étude sur le français, GuIMIER (1998) propose une représentation syntaxique pour les attributs de l'objet infinitifs, dans laquelle le verbe de perception sous-catégorise trois constituants et sélectionne deux arguments sémantiques, le sujet et un événement dénoté par le SN objet et l'infinitif; une interprétation à montée du sujet en position d'objet est ainsi privilégiée.

16 DeCLERCK (1982) envisage trois interprétations différentes possibles pour les constructions « NP + V-ING » à la suite d'un verbe de perception : 1) une lecture phrastique, où la séquence est la contrepartie progressive d'une séquence « NP + base verbale »;2) une interprétation où la proposition en -ING est un pseudo-modifieur comparable à une relative ; 3) une lecture où la proposition en -ING correspond à un adjoint prédicatif, qui peut être séparé du syntagme qui précède par une virgule. Les deux dernières interprétations ne sont pas sans rappeler les problèmes posés par les énoncés traditionnellement dits à attribut de l'objet.
} 
En effet, dans une configuration à $\mathrm{SN}+$ attribut de l'objet, ces deux constituants syntaxiques qui semblent autonomes correspondent à une seule proposition logique, un seul argument sémantique, mais il est plus délicat de dire qu'ils forment un seul constituant phrastique ${ }^{17}$.

Il existe donc une dépendance mutuelle entre les deux prédications : une dépendance syntaxique, dans le sens où l'attribut dépend indirectement du verbe recteur - dépendance médiée par la présence du syntagme nominal objet - et une dépendance sémantique inverse, la présence de l'attribut pouvant faire varier le sens originel du verbe recteur.

\section{Typologie des constructions à attribut de l'objet et comparaison des deux verbes see et voir}

On prêtera une attention particulière à trois paramètres primordiaux pour déterminer le sens véhiculé par l'énoncé dans son ensemble, avec les trois types d'attributs de l'objet sélectionnés pour cette étude (adjectival, prépositionnel et nominal) :

- la catégorie grammaticale de l'attribut de l'objet et sa relation avec l'objet ;

- le référent de l'objet, et sa nature ontologique ;

- la compatibilité sémantique entre l'attribut de l'objet et le verbe recteur.

\subsection{Syntagme adjectival attribut}

Les deux verbes retenus pour cette étude acceptent un syntagme adjectival attribut avec une fréquence assez élevée.

(7a) Molly's sleep had been deep and dreamless but she woke up early, saw Hugh unconscious beside her and replaced the sheet he had kicked away as she might cover one of the children. $(B N C)$

(7b) She knocked and opened the door and saw Patrick asleep in his wing chair. Ella considered daytime sleeping decadent. (BNC)

(7c) His tattoo had been done the previous winter by a Chinese man in Harlesden, who specialized in non-fade colours, fluorescents and airbrush fantasy. No one ever saw Jasper $\underline{\text { naked, }}$, since he skived off going swimming with his school and Tina was never present when he bathed, but he intended, one day, as a treat or reward, to show the tattoo to Bienvida. (BNC)

(7d) Finally, grabbing the bedcover and wrapping it round herself, she crept back to the head of the stairs. She'd seen Leo angry, mocking, bleak, but she had never seen him look so coldly murderous, so devoid of any human feeling, and she shivered. (BNC)

(7d') \# She'd seen that Leo was angry, mocking, bleak, [...].

(8a) Il se leva, et, dans la glace bleuâtre, il se vit plus pâle qu'à l'ordinaire. (Lextutor)

(8b) Cependant les portes de Notre-Dame étaient restées ouvertes, laissant voir l'église vide, désolée, en deuil, sans cierges et sans voix. (Lextutor)

(8c) Corinne s'étonna de la voir seule ainsi dans le jardin, et s'imagina que lord Nelvil ne tarderait pas à la rejoindre, et que peut-être il lui avait demandé un entretien secret, [...]. (Frantext)

\footnotetext{
${ }^{17}$ Nous avons, dans un travail récent (non publié), développé l'hypothèse d'un « syntagme nominal étendu » pour analyser les compléments non finis des verbes de perception et de cognition. Cette hypothèse pourrait être appliquée aux constructions traditionnellement appelées « attribut de l'objet », mais des recherches plus approfondies sont nécessaires pour en vérifier la pertinence et la validité.
} 
(8d) Chaque fois qu'il m'arrivait de lever les yeux sur lui, je le voyais tranquille et souriant au milieu d'un nuage, comme Jupiter au cinquième acte d'amphitryon. (Frantext)

(8c') \# Corinne s'étonna de voir qu'elle était seule ainsi dans le jardin, [...].

Ces énoncés avec attribut adjectival véhiculent généralement un sens de perception physiologique. Le référent de l'objet est appréhendé dans une situation ou dans un état physique passager décrit par l'adjectif : en (7d), par exemple, le référent de Leo est soit en colère, soit moqueur, soit encore morne en fonction des moments où l'expérient l'appréhende, de même que le percevant en (8d) appréhende à chaque perception un référent souriant. Le verbe ne subit pas de glissement sémantique vers la cognition, où il s'apparenterait à consider / considérer, par exemple (voir (7b), qui comporte le verbe consider dont le SN objet daytime sleeping a pour attribut l'adjectif decadent).

(9a) By the time White was 14, he was using and selling drugs. Even at that early age, he knew he was headed toward perdition: "I was actually seeing myself dead in a few years." $(C O C A)$

(9b) 'It seems likely that you were the last person to see Glynn alive, apart from his murderer, but at the moment I am more interested in what you can tell me about him - the sort of man he was - and about his friends and his enemies as far as you can.' (BNC)

(9a') \# "I was actually seeing that I would be dead in a few years."

(10a) Car enfin tu n'es pas faite pour vivre dans ce petit pays ridicule, et j'aimerais mieux te voir morte que mariée à un grec. (Frantext)

(10b) Mme Braux, dans sa stupeur de voir bien vivante celle qu'elle s'attendait à retrouver morte, n'osait pas même l'embrasser ; et son ventre énorme encombrait tout le palier, empêchant les autres d'avancer. (Lextutor)

(10c) [...] nous l'avons vu partir qu'avec du regret! Car il nous avait r'accoutumés à lui, ainsi que notre bon père lui-même, qui le voyant $\underline{\text { instruit }}$, aimait à passer le temps à converser avec lui sur toutes choses nouvelles ; [...]. [sic] (Frantext)

$\left(10 \mathrm{a}^{\prime}\right) \quad \#[\ldots] \mathrm{j}$ 'aimerais mieux voir que tu es morte que mariée à un grec.

$\left(10 c^{\prime}\right)[\ldots]$ notre bon père lui-même, qui voyant qu'il était instruit $[\ldots]$.

OLSSON (1976, également cité par WILLEMS \& DEFRANCQ, 2000) souligne qu'il existe un rapport entre la nature de l'adjectif et l'interprétation de la structure. Les adjectifs qui réfèrent à des propriétés stables ou permanentes de l'objet ont tendance à donner une orientation subjective à l'énoncé, qui transmet alors une opinion, une appréciation, alors que les adjectifs exprimant des propriétés moins stables ou temporaires sont cantonnés à l'expression d'une perception physiologique. Cela se vérifie en (7-8) où les adjectifs, majoritairement descriptifs et non évaluatifs, dénotent une qualité qui s'accorde avec le sens originel du verbe recteur, celui d'une appréhension physiologique du réel. Inversement, en (10c), l'état d'éducation dénoté par l'adjectif instruit, qui exprime une qualité permanente associée au référent de l'objet, ne peut être appréhendé par un simple contact visuel. Il ne s'agit pas uniquement de voir, mais de transmettre un constat de l'expérient fondé (probablement mais pas uniquement) sur une perception visuelle. La présence de l'attribut adjectival fait ainsi varier le sens du verbe et, dans ce cas, une paraphrase avec une complétive semble appropriée (10c'). En revanche, cette manipulation n'est pas adéquate lorsque le verbe exprime un sens de perception visuelle physiologique (ex. (7d', 8c', 9a', 10a')). Cependant, l'énoncé manipulé en (10c') 
présente un côté déclaratif constatif absent de l'énoncé originel, qui, lui, tend plutôt vers un sens évaluatif; il n'y a donc pas identité sémantique parfaite entre les deux configurations syntaxiques - attribut de l'objet adjectival et complétive. On constate également que cette similitude sémantique entre attribut adjectival et complétive est rarement tenable en anglais, mais moins singulière en français (cf. (8c')).

Néanmoins, bien qu'en (9-10) dead / mort et alive / bien vivante dénotent une qualité stable, permanente, aucun des énoncés n'exprime une opinion ou un jugement. En (9a, 10a), ce qui est à l'œuvre est l'imagination, le mind's eye. Ce qui prime dans ces énoncés, ce n'est pas tant l'état-même du référent (myself, Glynn / te, celle qu'elle s'attendait à voir morte) que le caractère surprenant, imprévu ou non désiré de cet état aux yeux de l'expérient : en $(9 b, 10 b)$, c'est le contraste entre alive et dead, ou inversement entre morte et bien vivante, qui capte l'attention du percevant (voir, par exemple, sa stupeur en (10b), qui signale la nature inattendue de la situation). Ceci est en accord avec le fait que, dans le réel, on ne peut percevoir que des changements d'état ou des états brefs, temporaires, un état étant généralement continu en soi et donc non perceptible dans le cadre d'une perception visuelle non prolongée.

Dans les énoncés à attribut adjectival, la nature animée ou inanimée du référent de l'objet n'influe donc pas sur le sens véhiculé par l'énoncé dans son ensemble. Il semble bien que d'une part la configuration syntaxique, et d'autre part le sémantisme de l'adjectif génèrent le sens de perception physiologique prédominant dans ce type de configuration. Dans les quelques énoncés qui comportent un adjectif dénotant une propriété permanente, le sens du verbe présente une nuance différente : il glisse vers l'imagination (ex. (9a, 10a)), qui peut être interprétée comme une représentation mentale volontaire, ou vers la découverte d'un état de fait concret (9b, 10b).

\subsection{Syntagme prépositionnel attribut}

Avec les verbes de perception visuelle, on distingue deux types de syntagmes prépositionnels: les syntagmes exprimant un sens "spatial" et les syntagmes introduits par la préposition as / comme.

L'attribut est introduit par une préposition exprimant une localisation littérale ou métaphorique, et les verbes see et voir se combinent avec une grande variété de prépositions :

- see: near (11a), with (11b), on (11c), in (11d) et at (11e). Les prépositions in et on semblent les plus fréquentes, mais on trouve aussi against, amongst, around, behind, beneath, round, under, within, etc. ${ }^{18}$;

- voir : à (12a), dans (12b-c), en (12d), sous (12e) et sans (12f). Les prépositions $\grave{a}$ et en paraissent les plus courantes, mais on trouve aussi auprès, autour, avec, de, derrière, sur, etc., ainsi que des locutions prépositionnelles (à côté de, au milieu de, aux environs de, en état de, etc.).

Les syntagmes prépositionnels en lien avec la spatialité peuvent renvoyer à une "position physique" (au sens large du terme) et/ou à une situation ou un état

${ }^{18}$ ANDERSSON (1985 : 172) propose d'autres exemples similaires à ceux en (11) : (50b) I've never seen her like this. 
temporaire, passager - autrement dit une position métaphorique - du référent de l'objet; aucune de ces deux significations n'est l'apanage de prépositions spécifiques ; voir, par exemple, la préposition dans en (12b-c) qui peut exprimer tantôt l'une, tantôt l'autre.

- "position physique" (au sens large du terme) du référent de l'objet : [11a-b],

- $\quad$ situation ou état temporaire, passager, de ce référent : [11c-e], [12c-f].

(11a) One of the men came back with a woman. 'Come along, dear. Time for your medical tests.' Then they saw Karen near the door. 'What are you doing here?' (BNC)

(11b) Anyone entering from outside would only have seen Jim with his feet up in front of the fire, reading a thriller. $(B N C)$

(11c) She said: 'One entire building is on fire. There must be hundreds of people living there.' a hotel porter said he saw people on fire running from the block. And a photographer told how he saw a girl of about six so severely burned that a fireman could not bring himself to treat her by dousing her with a hose. (BNC)

(11d) I've found that after nearly two years of grooming and bathing dogs, cute as they are, it's nice to see men in suits again. (COCA)

(11e) Whenever one of the family is seen responding to their dog's demands, he or she must pay $10 \mathrm{p}$ (or 50p if you really want to see motivation at work!) into the other's boxes. (BNC)

(11a') Then they saw Karen standing near the door.

(11c') A hotel porter said he saw people ablaze running from the block.

(12a) En entrant dans la chambre il vit Lucile à genoux, et la tête cachée dans le sein de sa mère ; $[. .$.$] (Frantext)$

(12b) Il ne pouvait supporter de voir Corinne dans les bras d'un autre, il frémissait en contemplant l'image de celle qu'il aimait ainsi privée de vie ; [...]. (Lextutor)

(12c) Je gardai la chambre pendant un mois. Or, un soir, mon père furieux de me voir dans cet état pour si peu, s'écria : "Qu'est-ce que ce sera donc quand tu auras de vrais chagrins, si tu perds ta femme, tes enfants !» (Lextutor)

(12d) À travers les broussailles entrecroisées sur lui, il vit le pont en flammes, la Tourgue rouge de la réverbération, et, par l'écartement de deux branches, il aperçut au-dessus de sa tête, de l'autre côté, sur le rebord du plateau, vis-à-vis du château brûlant et dans le plein jour de l'incendie, une figure hagarde et lamentable, une femme penchée sur le ravin. (Lextutor)

(12e) Ce Chouan ne donna pas la moindre marque d'émotion en se voyant sous la surveillance de ces hommes aussi redoutables par leur intelligence que par leur force corporelle. (Lextutor)

(12f) Me voyant sans habit, dans la rue, en décembre, la chose le toucha. (Frantext)

(12a') En entrant dans la chambre il vit Lucile qui était à genoux, la tête cachée dans le sein de mère ; [...].

(12d') À travers les broussailles entrecroisées sur lui, il vit le pont brûlant, [...].

(12f') Me voyant $\underline{n u}$, dans la rue, en décembre, la chose le toucha.

Avec ce type de syntagme prépositionnel (SP), l'énoncé véhicule généralement le même sens de perception physiologique qu'avec un attribut adjectival exprimant une propriété ou qualité temporaire ; on pourrait d'ailleurs substituer au syntagme prépositionnel un adjectif (11c', 12f'), une proposition finie - une relative prédicative ou dite de perception en français (12a'), qui s'emploie uniquement lorsque l'acte perceptif est concomitant avec le procès perçu - ou encore non finie - en -ING en anglais (11a') ou au participe présent en français (12d'). Dans tous les cas, l'énonciateur indique en discours la situation ou l'état dans lequel se trouve l'entité au moment de l'appréhension de la réalité extérieure. 
On note également, avec ce type de syntagme prépositionnel, un phénomène qui est plus fréquent en français qu'en anglais, où l'ordre des mots est plus contraint : le syntagme prépositionnel attribut précède le SN objet à droite du verbe recteur de ce fait ne sont pas concernés les énoncés où l'objet pronominal est placé, en français, à gauche du verbe de perception.

(13a) Into this happy image comes Snickers' electric catle prod. It zaps Hawk awake. With a howl, he spins up on the couch to see behind him Kaplan and the rest of the Candy Bars, except for Kit Kat. [sic] (COCA)

(13b) When he could again risk opening his eyes, he saw on the bed of cedar boughs his new hat. He bent over a second time and picked it up. The circle Pela had cut from the white fur poncho to make a hole for his head served as the top of the head covering. Its sides, however, were made of a deep black fur that felt like mink. He touched its softness with his fingers, stroked it against his cheek, and smelled the herbs with which Pela had treated the pelts. (COCA)

(13c) At a single stroke his strangeness had become an asset instead of a liability, especially with members of the Foreign Mission Society, who saw in him the living embodiment of all they were working for - the conversion, and hence the salvation, of the entire world! (COCA)

(13c') [...] members of the Foreign Mission Society, who saw him as the living embodiment of all they were working for - the conversion, and hence the salvation, of the entire world!.

(14a) Il tenait puissamment les rames dans ses deux poings. Il avait l'air doux. On voyait à sa ceinture un poignard, deux pistolets et un rosaire. (Lextutor)

(14b) À peine avait-il achevé sa dernière phrase, que l'hôte se sentit appliquer dans le dos un léger coup de manche de fouet, il se retourna brusquement, et vit derrière lui un petit homme $\underline{\text { trapu}}$, sorti sans bruit d'un cabinet voisin, et dont l'apparition avait glacé de terreur la grosse femme, le chef et son marmiton.

(14c) Enivré du charme de vivre auprès d'elle, du désir ardent d'y passer mes jours, absente ou présente, je voyais toujours en elle une tendre mère, une sœur chérie, une délicieuse amie, et rien de plus. (Lextutor)

(14d) Les maîtres de l'Inde voient dans les chemins de fer le moyen le plus efficace pour combattre le fléau qui décime périodiquement leurs millions de sujets : la famine. (Frantext)

$\left(14 \mathrm{c}^{\prime}\right)[\ldots]$ je la voyais / considérais toujours comme une tendre mère, une sœur chérie, une délicieuse amie, et rien de plus.

(14d') Les maîtres de l'Inde voient les chemins de fer comme le moyen le plus efficace pour combattre le fléau qui décime périodiquement leurs millions de sujets : la famine.

Les énoncés se répartissent en deux catégories :

1) Aucune différence de sens notable n'apparaît entre les deux structurations linéaires: $\mathrm{SN}$ objet $+\mathrm{SP}$ attribut ou $\mathrm{SP}$ attribut $+\mathrm{SN}$ objet. Cela concerne notamment les prépositions qui expriment une localisation spatiale: behind (13a), on (13b), à (14a), derrière (14b). La position en fin d'énoncé du SN objet est motivée par des principes de structuration de l'information, notamment les principes du poids relatif (end weight) et du focus final (end focus) :

- le SN objet comporte de nombreuses informations et a, de ce fait, une longueur et un poids syntaxiques certains (13a, 14a) ou, du moins, un poids syntaxique plus important que celui du SP attribut ;

- placer l'objet en fin d'énoncé permet de conserver une cohésion discursive étroite, notamment lorsqu'il y a, dans le co-texte droit, un développement sur le référent de l'objet ; voir, par exemple, la longue 
description de his new hat en (13b) ou les expansions sur le petit homme trapu en (14b).

2) On retrouve ces deux caractéristiques de structuration de l'information dans la seconde catégorie d'énoncés (cf. poids syntaxique de l'objet plus lourd que celui de l'attribut en (14c-d), et développement sur l'objet dans le co-texte droit en (13c)). Cependant, ces énoncés se distinguent des précédents en ce que le verbe recteur subit un glissement sémantique.

- l'énoncé véhicule un sens d'inférence perceptuelle, voire de cognition pure : l'idée qui se dégage est qu'une perception, visuelle ou autre, sert de fondement à une évaluation portée par l'expérient sur le référent du syntagme prépositionnel; en (13c), par exemple, le référent de him est considéré comme l'incarnation des valeurs pour lesquelles ils travaillaient. Une paraphrase avec le verbe de cognition consider/considérer est tout à fait appropriée dans ce contexte (cf. (13c', 14c')). C'est ce jugement, cette évaluation, qui passe au premier plan, plutôt que le sens de perception physiologique.

- la préposition n'a pas un sens spatial mais métaphorique en (13c, 14c-d), ce qui favorise cette lecture, bien que les prépositions concernées dans les trois énoncés en question ne figurent pas uniquement dans ce type d'énoncé (voir dans en (12b), par exemple, qui exprime une localisation spatiale).

- il est possible de gloser ces énoncés avec un autre type de syntagme prépositionnel, introduit par as / comme. Dans les énoncés manipulés en (13c', 14c'-d'), l'ordre des constituants est inversé et l'objet des énoncés originels se trouve en position de complément de la préposition comme, mais le sens reste inchangé, comme c'est le cas avec certains énoncés comportant as / comme.

Le second type de syntagme prépositionnel est introduit par as ou comme, configuration très fréquente avec les verbes à l'étude. Comme pour le premier type de syntagmes prépositionnels étudié ci-dessus, deux sous-catégories d'énoncés peuvent être distinguées :

(15a) In what ways, then, did the patient's psychical blindness manifest itself? He could not see objects as unified, self-contained, and organized figures, as a person does with normal vision. (Brown)

(15b) I almost wish now that I had settled for chronic asthma with which to punish Miller, dispensing with the limp and the sausage fingers altogether. Asthma has a great many things in its favour so far as my present purpose is concerned. I took at least two hours before reluctantly passing it over. This illness can be frightening in its sudden, panting melodrama, outward struggle of a diseased bronchial tree. I played around with these harsh gasps for a while, seeing Miller $\underline{a s}$ a big pink fish stranded on a cobble-stoned beach, letting broken scraps of dialogue decorate the page. But, alas, the printed word cannot adequately convey the panting, gasping misery of this particular torment. $(B N C)$

(15a') He could not see objects constituting unified, self-contained, and organized figures.

(16a) Mais le meuble que je vois et sens comme réalité concrète oppose son immobilité et sa résistance à la mobilité de mon être. (Lextutor)

(16b) Les compagnies de Bleus appartenant à la garnison de Mortagne et qui avaient accompagné cette horrible voiture jusqu'aux limites de leur étape, où Hulot était venu les 
remplacer dans ce service, à juste titre nommé par ses soldats une scie patriotique, retournaient à Mortagne et se voyaient dans le lointain comme des points noirs. (Lextutor)

(16c) Et voilà ! C'est tout. Oui. Tu ne les vois plus. Pardon, je les vois encore comme $\underline{a m i}$; nous n'avons pas rompu tout fait. [sic] (Lextutor)

(16b') [...] retournaient à Mortagne et se voyaient dans le lointain représenter / représentant des points noirs.

Une première catégorie d'énoncés véhicule elle aussi un sens de perception physiologique (15-16) : le référent de l'objet est une entité perceptible (et perçue) au moyen de la vue dans le réel. Une manipulation avec proposition non finie en ING / au participe présent (15a'; 16b') est donc appropriée dans les deux cas. En (15a), on déduit du co-texte qu'une perception visuelle déficiente (the patient's psychical blindness) - et par conséquent non effective - rend le patient incapable d'appréhender les objets tels qu'ils sont vraiment. Au contraire, en (16a), l'objet « meuble » est effectivement bien perçu comme appartenant à la réalité. Le sens véhiculé dans ces énoncés équivaut alors à « voir X comme étant... / en tant que », car il existe un rapport concret référentiel entre l'entité perçue et la qualification qui lui est attribuée par le groupe nominal au sein du syntagme prépositionnel. Le référent de l'objet est classé dans une catégorie, une classe, dénotée par le groupe nominal complément de la préposition as / comme, et cette catégorisation n'est pas problématique en ce qu'elle constitue une propriété objective du référent de l'objet : elle pourrait être faite par tout énonciateur, tout membre de la communauté linguistique (voir $(15 \mathrm{a}, 16 \mathrm{a})$ et la qualification attribuée à objets / meuble) ou, du moins, elle a déjà été posée dans le contexte. L'énoncé $(15 b)$ semble se détacher cependant, et se rapprocher de ceux présents en (17-18) : l'appréhension dans le même temps du référent de Miller et de la qualification qui lui est attribuée (a big pink fish stranded on a cobble-stoned beach) relève plutôt d'une visualisation mentale, voire de l'imagination, que d'une véritable perception.

Les énoncés de la seconde catégorie (17-18) véhiculent, quant à eux, un sens d'inférence perceptuelle ou de cognition. Le syntagme prépositionnel ${ }^{19}$ dénote une caractéristique attribuée au référent de l'objet après l'exercice du jugement subjectif de l'expérient ; celui-ci peut être effectué sur la base de données perceptuelles $(17 \mathrm{a}, 18 \mathrm{a})$ - d'où le sens d'inférence perceptuelle - ou non $(17 \mathrm{~b}, 18 \mathrm{~b}-\mathrm{c})$ - d'où le sens de cognition.

(17a) Charlemagne and his wife, from a ninth-century manuscript. In retrospect, we can see Charles as a man of immense energy; we know from chronicles that he slept lightly and that he commenced state work very early in the morning, judging litigants in his private chamber. $(B N C)$

(17b) However, in this chapter, I do not want to tackle any of those perceptions head on. Instead, I want to try to develop a further view of what it means to see criticism as a significant feature of higher education. (BNC)

(17a') In retrospect, we can see that Charles was a man of immense energy; [...].

\footnotetext{
${ }^{19}$ Les prépositions as et comme peuvent prendre d'autres types de compléments qu'un SN : voir, en (18c), le syntagme prépositionnel en parallèle; on peut également trouver un adjectif ou une gérondive en anglais, et un participe présent en français.
} 
(18a) Non, comme de nombreux militants de mouvement, il se voit plutôt comme un syndicaliste de la fin du XIXe siècle, [...]. (Lextutor)

(18b) Et peu à peu, l'existence, elle se mettait à la voir, en sortant du livre, avec la désillusion d'un retour de cimetière, à la voir comme un passage, une route à traverser en voyageur et en étranger qui ne fait que toucher en chemin l'inanité, la vacuité, la vanité des vanités de toutes les choses hors de Dieu. (Frantext)

(18c) $[\ldots]$ mais pour en être tout à fait certains, il nous suffit de nous rendre un compte exact de la signification de tous ces termes. Je vois d'abord comme en parallèle et presque en opposition l'entendement et la volonté. (Frantext)

(18a') [...] il se considère plutôt comme un syndicaliste de la fin du XIXe siècle, [...].

(18c') Je vois / considère d'abord que l'entendement et la volonté sont en parallèle et presque en opposition.

Le verbe a alors un sens proche de celui de consider, realise, en anglais ou de considérer, se rendre compte, en français. Les facultés perceptives font place aux facultés mentales de jugement, et l'on constate que le référent de l'objet ne peut pas - toujours - être perçu par des organes sensoriels dans le réel : la critique (17b), pas plus que l'entendement et la volonté (18c), ne peuvent faire l'objet d'une appréhension perceptive, mais uniquement intellectuelle. De même, en (17a), il ne s'agit pas de voir Charlemagne, décédé au moment de "perception" (cf. in retrospect) ; cependant, une perception visuelle est à l'œuvre (cf. from a ninthcentury manuscript, from chronicles) et cette lecture sert de fondement à une appréciation (a man of immense energy) portée par l'expérient sur Charlemagne, référent de l'objet. La même lecture s'applique à (18a), l'identification à un syndicaliste d'un siècle passé ne pouvant se faire qu'après évaluation et comparaison a posteriori entre les actions menées par deux référents animés humains.

Le verbe recteur exprime ainsi un constat ou une opinion de l'expérient non plus sur une seule entité, mais sur un état de fait dans son ensemble; c'est ce que démontre également la possibilité d'une substitution par une complétive, très souvent appropriée dans ces occurrences $\left(\left(17 \mathrm{a}^{\prime}, 18 \mathrm{c}^{\prime}\right)\right.$ - à noter qu'en $(18 \mathrm{a})$, on utilisera plutôt le verbe considérer avec une construction à attribut de l'objet également). Contrairement aux énoncés en (15-16), l'assignation du référent de l'objet dans une catégorie spécifique résulte de l'exercice du jugement de l'expérient. Comme la qualification attribuée n'est pas nécessairement partagée par tous, et peut n'être valable que pour l'expérient (ex. l'identification à un syndicaliste de la fin du XIX ${ }^{\mathrm{e}}$ siècle en (18a)), l'énoncé exprime une opinion par conséquent hautement subjective. Cette subjectivité de la qualification identificatoire provient du complément de la préposition, qui exprime une évaluation (ex. voir le sens de l'adjectif dans a significant feature en (17b)). Le syntagme prépositionnel en as / comme exprime une évaluation de propriétés, qui conduit à identifier le référent de l'objet, par le biais de la préposition, avec les propriétés évoquées dans le SN complément prépositionnel. Cette évaluation va de pair avec l'expression d'un jugement ou d'une opinion subjective de la part de l'expérient, faisant alors glisser le sens originel du verbe. En effet, le lien entre les propriétés du référent de l'objet et les qualités ou propriétés évoquées par le syntagme prépositionnel n'est pas évident pour tous. 
Ainsi, avec les verbes de perception, la construction avec attribut prépositionnel se prête à trois types de lecture, selon trois paramètres distincts: la nature perceptible ou non du référent de l'objet; la compatibilité sémantique de l'attribut prépositionnel avec la modalité du verbe recteur ; le lien - évident ou subjectif entre le référent de l'objet et les propriétés évoquées par ce qui suit la préposition, as / comme notamment.

\subsection{Syntagme nominal attribut}

Les syntagmes nominaux constituent le dernier type d'attribut de cette étude car ils sont, de manière générale, moins fréquents en attribut de l'objet que leurs contreparties adjectivales ou prépositionnelles ${ }^{20}$. Cela peut s'expliquer par le fait qu'un syntagme nominal indique d'emblée l'appartenance à une catégorie ; or, il semble difficile, dans le réel, d'appréhender une entité tout en lui attribuant une propriété qui la ferait appartenir à une classe précise autre que celle à laquelle elle appartient de façon inhérente - en d'autres termes, à celle dénotée par le SN objet. Cette catégorisation ou classification suppose en effet, de la part de l'expérient, une opération cognitive plus complexe : une perception physiologique combinée à un jugement mental immédiat. En revanche, les syntagmes adjectivaux et prépositionnels signalent l'attribution d'une qualité ou d'une position, spatiale ou métaphorique, fondée sur des données perceptuelles.

Dans cette configuration, l'attribut nominal en anglais présente un caractère gradable, condition sine qua non pour qu'il puisse instancier le site d'attribut de l'objet. C'est souvent la présence d'un adjectif au sein du SN attribut qui permet à celui-ci d'exprimer une gradation, de renvoyer à une notion dont les propriétés peuvent être évaluées : en (19a), par exemple, la qualification par les adjectifs dangerous et hostile confère une gradabilité au SN attribut, et la suppression de ces deux éléments engendre un énoncé peu acceptable (19a'). En (19c), au contraire, le référent de you est identifié au centre attracteur du domaine notionnel, c'est-à-dire au prototype par excellence de la notion, à l'occurrence imaginaire qui possède toutes les propriétés de la notion /anti-war activist/ ou /global warming activist/ au plus haut degré.

(19a) Gerald watched Gudrun closely, whilst she repulsed Hermione. There was a body of cold power in her. He watched her with an insight that amounted to clairvoyance. He saw her a dangerous, hostile spirit, that could stand undiminished and unabated. (Women in Love, D.H. Lawrence)

(19b) 'From this moment, Spanish Americans, you see yourselves free men.... Your destiny no longer depends on minister, viceroys or governors; they are in your own hands.' $(B N C)$

(19c) 'No, it's that you're lecturing me about global warming. It's that you're lecturing every time, Sally Field said it best. "Oh, well, I'll say it again". Well, that's great. But I like Sally Field. I really like you. And why is it these people assault $50 \%$ of the movie-going audience? Please, I, I want to watch you perform character roles. But that when you're on TV, I see you the anti-war or the global warming activist, and I can't separate it anymore. So now, instead of "I'm the king of the world", I see, "And we should all be driving Priuses." That's what I see. (COCA)

(19d) Lance was a bitter disappointment to Marquette, who, as the owner of a sporting goods store, saw himself the gatekeeper to a young man's paradise. (COCA)

${ }^{20} \mathrm{Ceci}$ est également vrai pour les syntagmes nominaux attributs du sujet avec un verbe de perception copule en anglais (ex. look, sound). 
(19a') ?? He saw her $\underline{\text { a spirit, }}$, that could stand undiminished and unabated.

(19a") He saw her as a dangerous, hostile spirit, that could stand undiminished and unabated.

(19b') \#? From this moment, Spanish Americans, you see that you are free men...

(19c') \#? But that when you're on TV, I see that you are the anti-war or the global warming activist $[\ldots]$.

(20a) Des caprices inconnus ou la passion donnèrent à cette figure des couleurs étincelantes, et l'on vit que le monde entier ne devait plus être rien pour cette jeune fille du moment où elle y distinguait une créature ; mais tout à coup elle rentra dans un calme forcé en $\underline{\text { se }}$ voyant, comme un acteur sublime, l'objet des regards de tous les spectateurs. (Lextutor)

(20b) Quand les Goths ravagèrent la Grèce, toutes les Bibliothèques ne furent sauvées du feu que par cette opinion semée par l'un d'entre eux, qu'il falloit laisser aux ennemis des meubles si propres à les détourner de l'exercice militaire, \& à les amuser à des occupations oisives \& sédentaires, Charles VIII se vit maître de la Toscane $\&$ du Royaume de Naples sans avoir presque tiré l'épée ; [...]. (Lextutor)

(20c) En certains pays, quand un cultivateur se voit père d'une fille, il plante un petit bois qui grandit avec l'enfant, et fournit sa dot au moment où elle se marie. (Frantext)

(20d) Fillette, je désirais devenir maîtresse d'école. Quant à ma mère, elle me voyait plutôt pharmacienne. Vous connaissez le résultat. (Lextutor)

$\left(20 a^{\prime}\right) \quad[\ldots]$ elle rentra dans un calme forcé en voyant qu'elle était l'objet des regards de tous les spectateurs.

$\left(20 c^{\prime}\right) \quad[\ldots]$ quand un cultivateur se rend compte / constate qu'il est père d'une fille, [...].

Les énoncés véhiculent ici un sens d'inférence perceptuelle : l'appréciation ou le jugement porté par l'expérient est fondé sur des données perceptuelles. En (19a), l'observation (Gerald watched Gudrun) conduit le percevant à porter un jugement sur l'entité perçue ; une substitution en as serait d'ailleurs appropriée en (19a').

On retrouve cette évaluation dans certains énoncés en (20), (20a-b) notamment : l'évaluation ou induction conclusive l'objet des regards de tous les spectateurs en (20a) ne peut être issue que d'une observation visuelle. En revanche, ce qui transparaît nettement grâce aux énoncés (20), c'est la différence de distribution entre see et voir : en effet, des énoncés tels qu'en (20b-d) ne sauraient correspondre à des configurations avec see suivi d'un $\mathrm{SN}+$ attribut nominal, pas plus que d'un $\mathrm{SN}+$ attribut prépositionnel en as - contrairement aux énoncés en (19). Ainsi, une paraphrase avec complétive n'est pas la plus appropriée pour les énoncés en anglais (19b', d'), car le sens de perception physiologique reste sous-jacent, même si le verbe see vient à signifier consider. Inversement, une complétive en que paraît adéquate pour véhiculer la déduction ou le constat exprimé par les énoncés en français (20a', c'); d'ailleurs la paraphrase en (20c') comporte le verbe constater / se rendre compte, plus adapté que le verbe considérer dans ces énoncés, car il ne s'agit pas de porter une appréciation ou un jugement sur le référent de l'objet. Néanmoins, si, comme mentionné plus haut, une complétive véhicule sans conteste un sens déclaratif constatif, la configuration en (20a-c), dans laquelle voir est employé avec un pronom réfléchi réflexif objet $(\mathrm{se})$, combine l'expression à la fois d'une expérience perceptive et d'une inférence fondée sur des données perceptuelles, subjectives par nature ; de ce fait, celles-ci semblent plus susceptibles d'être contestées qu'une opinion exprimée par le biais d'une complétive en that / que, qui signalerait un constat acquis après un processus de réflexion. (20d) semble ici faire exception, en ce qu'il véhicule une "perception" imaginaire qui 
porte sur un état de fait (pharmacienne) encore virtuel, ce qui contribue à donner un sens d'éventualité à l'énoncé.

Il apparaît que la proximité de l'objet et de l'attribut dans la linéarité du discours reflète l'association immédiate, pour l'expérient, entre le référent de l'objet et la catégorie notionnelle dénotée par le syntagme nominal - voir, à titre d'exemple, l'absence d'article dans le SN attribut dans les énoncés (20b-d) ${ }^{21}$. Cette immédiateté du jugement pourrait expliquer pourquoi les attributs nominaux sont peu fréquents avec les verbes de perception. Comme le langage est, selon nous, iconique des processus cognitifs à l'œuvre dans le réel, ceux-ci doivent être marqués dans un énoncé où le verbe de perception entre dans une configuration à attribut de l'objet : la perception physiologique, l'inférence qui s'ensuit et l'évaluation qui en découle. Le processus inférentiel et le jugement qui s'ajoutent à la perception physiologique sont alors souvent exprimés en discours par la préposition as / comme qui, en tant que relateur, signale la relation qui existe entre le processus perceptif et l'appréciation ou le jugement qui est fondé sur des données perceptuelles ${ }^{22}$.

\section{Synthèse}

Avec see et voir, les énoncés avec attribut de l'objet peuvent appartenir à trois catégories sémantiques, pour lesquelles les manipulations avec d'autres types de constituants se sont avérées utiles :

1) sens de perception physiologique ; glose avec une proposition non finie ;

2) sens d'inférence perceptuelle; glose avec une complétive en that / que;

3) sens de cognition; glose avec une complétive en that / que.

\footnotetext{
${ }^{21}$ Les énoncés en français (20b-d), mais aussi (10a), ou encore les énoncés en anglais tels que (15b), présentent un fonctionnement et des propriétés similaires à ceux des constructions causatives ou résultatives. Il serait pertinent de mener une étude comparative approfondie de ces deux types de structures dans des recherches ultérieures.

${ }^{22}$ Des énoncés tels que le suivant n'ont pas été étudiés dans cette section : Le groupe de tire-laine, à partir de ce moment, le voyant la tête en l'air et bouche bée, se rapproche de lui. (Frantext). En effet, bien que le segment souligné en italiques se présente sous la forme d'un syntagme nominal, il s'interprète davantage comme un syntagme prépositionnel tel que ceux qui ont été étudiés dans la première section de la partie 3.2 (ex. le voyant avec la tête en l'air et la bouche bée).
} 


\begin{tabular}{|c|c|c|c|}
\hline Attribut adjectival & \multicolumn{2}{|c|}{ Attribut prépositionnel } & Attribut nominal \\
\hline \multicolumn{4}{|c|}{ Verbe anglais see } \\
\hline $\begin{array}{l}\text { - adjectif de qualité } \\
\text { temporaire : perception } \\
\text { physiologique } \\
\text { - adjectif de qualité } \\
\text { "permanente": perception } \\
\text { physiologique, imagination }\end{array}$ & $\begin{array}{l}\begin{array}{l}\text { - SP de sens } \\
\text { spatial }= \\
\text { perception } \\
\text { physiologique } \\
\multicolumn{1}{c}{\text { - SP en }} \\
\text { as }= \\
\text { identification } \\
\rightarrow \text { perception } \\
\text { physiologique }\end{array}\end{array}$ & $\begin{array}{l}\text { - SP en } a s=\text { inférence } \\
\text { perceptuelle ou } \\
\underline{\text { cognition }}\end{array}$ & $\begin{array}{l}\text { - inférence } \\
\text { perceptuelle }\end{array}$ \\
\hline \multicolumn{4}{|c|}{ Verbe français voir } \\
\hline $\begin{array}{l}\text { - adjectif de qualité } \\
\text { temporaire : perception } \\
\text { physiologique } \\
\text { - adjectif de qualité } \\
\text { "permanente": perception } \\
\text { physiologique } \\
\text { ou cognition }\end{array}$ & $\begin{array}{l}\begin{array}{l}\text { - SP de sens } \\
\text { spatial }= \\
\text { perception } \\
\text { physiologique } \\
\text { - SP en }\end{array} \\
\begin{array}{l}\text { comme }= \\
\text { identification } \\
\rightarrow \text { perception } \\
\text { physiologique }\end{array}\end{array}$ & $\begin{array}{l}\text { - SP en comme }= \\
\text { inférence perceptuelle } \\
\text { ou cognition }\end{array}$ & $\begin{array}{l}\text { - inférence } \\
\text { perceptuelle } \\
\text { - constat } \\
\text { - éventualité }\end{array}$ \\
\hline
\end{tabular}

\begin{tabular}{|c|c|c|}
\hline Perception physiologique & Inférence perceptuelle & Cognition \\
\hline \multicolumn{3}{|c|}{ Verbe anglais see } \\
\hline $\begin{array}{l}\text { - attribut adjectival - adjectif } \\
\text { de qualité temporaire } \\
\text { - attribut prépositionnel avec } \\
\text { sens spatial } \\
\text { - attribut prépositionnel en } \\
\text { as avec sens d'identification }\end{array}$ & $\begin{array}{l}\text { - attribut prépositionnel en as } \\
\text { - attribut nominal - le SN dénote un } \\
\text { jugement, l'évaluation d'un degré }\end{array}$ & $\begin{array}{l}\text { - attribut adjectival - } \\
\text { adjectif de qualité } \\
\text { "permanente" } \\
\text { (imagination) } \\
\text { - attribut prépositionnel en } \\
\text { as }\end{array}$ \\
\hline \multicolumn{3}{|c|}{ Verbe français voir } \\
\hline $\begin{array}{l}\text { - attribut adjectival - adjectif } \\
\text { de qualité temporaire } \\
\text { - GN + attribut } \\
\text { prépositionnel avec sens } \\
\text { spatial } \\
\text { - attribut prépositionnel en } \\
\text { comme avec sens } \\
\text { d'identification }\end{array}$ & $\begin{array}{l}\text { - attribut prépositionnel en comme } \\
\text { - attribut nominal - le SN dénote un } \\
\text { jugement, l'évaluation d'un degré }\end{array}$ & $\begin{array}{l}\text { - attribut adjectival - } \\
\text { adjectif de qualité } \\
\text { permanente (imagination, } \\
\text { constat) } \\
\text { - attribut prépositionnel en } \\
\text { comme } \\
\text { - attribut nominal (constat, } \\
\text { éventualité) }\end{array}$ \\
\hline
\end{tabular}

On note que le verbe français a des significations cognitives plus étendues et plus fréquentes que sa contrepartie anglaise. Cette différence dans les emplois modernes de voir et see pourrait être le reflet de la distribution déjà différente de leurs "ancêtres" respectifs. See provient du vieil anglais (ge)séon, qui signifiait «to see, look, behold; observe, perceive, understand; experience, visit, inspect » $(O n E D)$. Ainsi, si son évolution vers le registre de la cognition transparaît dès son origine, ses significations sont essentiellement centrées sur l'expérience. Concernant voir, son étymologie remonte au latin videre, dont les significations 
étaient multiples : «percevoir par la vue, être témoin de, remarquer, constater », mais aussi «voir par la pensée ou l'imagination, juger, examiner, déterminer » (TLFi). Les emplois cognitifs restent répandus en français contemporain, sans être nécessairement liés à une expérience visuelle. En revanche, cette expérience visuelle ou plus généralement perceptive - reste sous-jacente avec see, qui reste proche du sens et de la distribution de (ge)séon en vieil anglais. De ce fait, une glose avec complétive convient plus naturellement à voir qu'à see; comme le souligne MULLER (2000), elle s'applique tout aussi naturellement aux verbes de croyance, tels que savoir, présumer, qu'aux verbes psychologiques.

Reste à évoquer la différence entre les trois types d'attributs. L'utilisation d'un syntagme prépositionnel en as/comme peut indiquer une identification ou qualification subjective effectuée par l'expérient, subjectivité qu'on ne trouve pas avec les syntagmes adjectivaux et prépositionnels de sens spatial. Ceux-ci tendent à donner un caractère plus objectif à l'appréciation qui est portée sur le référent de l'objet. L'adjectif signale que l'acte perceptif permet de voir instantanément (dans le réel ou dans son esprit) une qualité ou propriété du référent de l'objet, tandis qu'avec as et comme, celle-ci est attribuée au terme d'une comparaison identificatoire. La perception immédiate de la situation et/ou des qualités de l'entité contraste avec l'inférence déductive plus longue signalée par le syntagme en as : adjectif et syntagme prépositionnel en as auraient alors une structure iconique de l'écart qui existe (ou non) entre appréhension perceptive et appréciation subjective fondée sur des données perceptuelles. On retrouve cette proximité linéaire avec les attributs nominaux, mais leur fréquence moindre avec les verbes de perception reflète la difficulté à percevoir et, dans le même instant, à évaluer un degré ou classer dans une catégorie de propriété gradable.

On constate donc que le sens véhiculé par l'ensemble de l'énoncé ne tient pas uniquement à la configuration syntaxique avec attribut de l'objet; il dépend également de la relation référentielle entre le verbe recteur et l'objet, plus précisément à la relation sémantique qui existe entre le procès et le référent de l'objet, ainsi qu'à la nature perceptible ou non de ce référent, sans oublier le lien objectif ou subjectif entre ce référent et la description dénotée par l'attribut. Dans les énoncés à lecture d'inférence perceptuelle et de cognition, l'attribut apporte une contribution tant syntaxique que sémantique en ce qu'il fait varier le sens du verbe recteur.

Pour finir, qu'est-ce qui motive le choix, en discours, entre un verbe de perception et un verbe de cognition, tel que consider, realise, regard ou bien considérer, constater, lorsque les énoncés véhiculent un sens d'inférence perceptuelle ou de cognition?

- Avec un verbe de perception, l'énoncé véhicule à la fois évidentialité et point de vue. Le verbe de perception indique la source évidentielle - visuelle ici sur laquelle se fonde l'inférence, et dont découle l'appréciation, le jugement porté par l'expérient. La perception visuelle sert de caution à l'appréciation portée, qui est ainsi présentée comme étant plus objective puisqu'elle est inférée d'indices perceptifs. Ainsi, le verbe de perception fonctionne comme un évidentiel ${ }^{23}$ : il

23 «Évidentiel» s'entend comme marquant la source de l'information (voir AIKHENVALD, 2004). 
véhicule l'impression d'une vérité plus objective et plus généralement admise qu'avec consider, par exemple. L'emploi d'un verbe de perception signale que le commentaire effectué est fondé sur des données perceptuelles.

- En revanche, avec un verbe de cognition, l'énoncé véhicule seulement le point de vue, l'opinion de l'expérient; il ne fait généralement pas mention d'une quelconque source évidentielle : l'énoncé indique rarement sur quels indices est basée l'opinion transmise... probablement parce qu'elle ne provient généralement pas de faits objectifs. L'opinion ou le jugement ainsi présenté paraît alors plus subjectif qu'avec un verbe de perception, l'énoncé relevant alors davantage de l'épistémicité.

\section{Conclusion}

Il apparait, au terme de cette étude, que le champ d'application sémantique des deux verbes prototypiques de la perception visuelle see et voir est très étendu. Peutêtre faut-il en chercher la raison dans les processus cognitifs à l'œuvre dans le réel : en effet, la perception est d'une part l'appréhension du monde extérieur par un expérient, mais elle est également la source de toute connaissance. Le glissement sémantique de see et voir vers l'inférence perceptuelle puis la cognition n'est donc guère surprenant, et semble être iconique des processus cognitifs à l'œuvre dans la réalité : de la perception vers la connaissance. Dans le même ordre d'idée, les structures du langage semblent être le reflet de ces processus cognitifs. La configuration avec attribut de l'objet est une construction seconde, qui traduit un processus cognitif mais aussi énonciatif plus complexe que celui qu'exprime un simple syntagme nominal objet, qu'il s'agisse de préciser la situation ou l'état du référent de l'objet, ou bien d'exprimer un état de fait à son sujet.

Les énoncés à l'étude véhiculent trois grandes significations différentes, allant de la perception physiologique à la cognition, en passant par l'inférence perceptuelle. Il est donc préférable de concevoir un continuum entre ces trois grandes catégories plutôt qu'une juxtaposition de catégories étanches; c'est d'ailleurs ce qu'invite à faire la présence de structures identiques dans deux ou trois types différents de catégories sémantiques. Le glissement sémantique des verbes de perception, preuve de leur polyvalence et de leur plasticité, découle d'une combinaison de plusieurs facteurs : souplesse syntaxique et sémantique, catégorie syntaxique des compléments et configuration syntaxique, liens sémantiques entre verbe et complément, mais aussi entre les compléments. Ainsi, la simplicité ou la complexité du compte rendu, et donc du discours, semble refléter celle des processus perceptifs et cognitifs dans le réel.

\section{BIBLIOGRAPHIE}

AARTS Bas (1995), Secondary predicates in English, in : AARTS Bas; MEYER Charles F. (éd.), The Verb in Contemporary English. Theory and Description, Cambridge, Cambridge University Press, p. 75-101.

AIKHENVALD Alexandra (2004), Evidentiality, Oxford, Oxford University Press. ANDERSSON Evert (1985), On Verb Complementation in Written English, Lund, CWK Gleerup. 
CROFT William (1991), Syntactic categories and grammatical relations, Chicago/London, The University of Chicago Press.

Culioli Antoine (1990), Pour une linguistique de l'énonciation, Tome 1, Opérations et représentations. Gap / Paris, Ophrys.

DECLERCK Renaat (1981), Pseudo-Modifiers, Lingua 54(2-3), p. 135-163.

DECLERCK Renaat (1982), The Triple Origin of Participial Perception Verb Complements, Linguistic Analysis 10(1), p. 1-26.

GROUSSIER Marie-Line; RIVIÈRE Claude (1996), Les mots de la linguistique. Lexique de linguistique énonciative, Paris, Ophrys.

GUIMIER Emilie (1998), Les constructions à prédicat de l'objet des verbes de perception, in : FORSGREN Mats, JONASSON Kerstin, KRONNING Hans (éd.), Prédication, assertion, information. Actes du colloque d'Uppsala, Uppsala, Acta Universitatis Upsaliensis, p. 231-241.

HAVU Eva ; PIERRARD Michel (2008a), Réduction et intégration de prédications : paramètres pour l'analyse des co-prédications adjectivantes, Discours 2, URL : $<$ http://discours.revues.org/1042>.

HAVU Eva ; PIERRARD Michel (2008b), La prédication seconde en français : essai de mise au point. Travaux de linguistique 57, p. 7-21.

LACASSAIN-LAGOIN Christelle (2007), La complémentation des verbes de perception en anglais contemporain. Forme et sens des comptes rendus de perception directe et indirecte. Thèse de doctorat non publiée, Pau, Université de Pau et des Pays de l'Adour.

LACASSAIN-LAGOIN Christelle (2011), Les comptes rendus de perception avec attribut de l'objet. Quelle intégration de l'altérité ?, in : CHINI Danielle, MARIELAVERROU Florence, PARSONS Michael (éd.), Intégration de l'altérité : formes et procédures. Regards croisés sur les aires anglophones et hispanophones, RIVS 5, Paris, L'Harmattan, p. 11-19.

LACASSAIN-LAGOIN Christelle (à paraître). Verbes de cognition et verbes de perception : étude comparée de la construction à attribut de l'objet, Corela.

LAMBRECHT M. Knud (2000), Prédication seconde et structure informationnelle : la relative de perception comme construction présentative, Langue française 127, Paris, Larousse, p. 49-66.

LYONS John (1968), Introduction to Theoretical Linguistics, London / Cambridge, Cambridge University Press.

MULLER Claude (2000), Les constructions à adjectif attribut de l'objet, entre prédication seconde et complémentation verbale, Langue française 127, Paris, Larousse, p. 21-35.

OLSSON Kerstin (1976), La construction verbe + objet direct + complément prédicatif en français: aspects syntaxiques et sémantiques, Stockholm, Université de Stockholm.

PALMER Frank R. (1988), The English Verb (2 ${ }^{\text {nd }}$ ed.), London, Longman.

PIERRARD Michel ; HAVU Eva (2014), L'Attribut de l'objet, une complémentation nucléaire ?, Travaux de linguistique 68, p. 27-48.

QUIRK Randolph ; GREENBAUM Sidney ; LEECH Geoffrey ; SVARTVIK Jan (1985), A Comprehensive Grammar of the English Language, London, Longman. 
Riegel Martin; Pellat Jean-Christophe; Rioul René (1994), Grammaire méthodique du français ( $5^{\mathrm{e}}$ éd.), Paris, Presses Universitaires de France.

VendLer Zeno (1967), Verbs and Times, in: Linguistics in Philosophy, Ithaca / London, Cornell University Press, [1957], p. 97-121.

Willems Dominique ; DEFrANCQ Bart (2000), L'attribut de l'objet et les verbes de perception, Langue française 127, Paris, Larousse, p. 6-20.

WiLliams Edwin S. (1983), Against Small Clauses, Linguistic Inquiry 14(2), p. 287-308.

\section{DICTIONNAIRES}

Le Trésor de la Langue Française informatisé (2001), Paris, Éditions du Centre National de la Recherche Scientifique, URL: < http://atilf.atilf.fr/tlf.htm >. (TLFi).

Online Etymology Dictionary (2001-2016), Douglas Harper (éd), URL : $<$ www.etymonline.com $>($ OnED).

The Oxford English Dictionary (2014) ( $2^{\text {nd }}$ ed.), Édition numérique, Oxford, Oxford University Press. URL : <www.oed.com >. (OED)

Le Dictionnaire de l'Académie française. (1992). (9éd.); URL: $<$ http://atilf.atilf.fr/academie9.htm $>$.

\section{CORPORA}

British National Corpus, Oxford University Press; URL: < http://corpus.byu.edu/bnc/> ; consulté le 22/04/2017 (BNC).

Brown Corpus, H. Kucera \& W. N. Francis (Brown).

The Corpus of Contemporary American English, M. Davies; URL : $<$ http://corpus.byu.edu/coca/ > ; consulté le 22/04/2017 (COCA).

Base textuelle FRANTEXT, ATILF - CNRS \& Université de Lorraine ; URL : $<w w w$.frantext.fr $>$; consulté le 22/04/2017 (Frantext).

Lextutor, Concordancier-corpus français (v.6.5); URL: <www.lextutor.ca/>; consulté le 22/04/2017 (Lextutor). 